Monatsschr Kinderheilkd 2015 • 163:517-520

DOI 10.1007/s00112-015-3316-9

Online publiziert: 23. Mai 2015

(c) Springer-Verlag Berlin Heidelberg 2015

K.-P. Zimmer

Abt. Allgemeine Pädiatrie \& Neonatologie, Zentrum für Kinderheilkunde und Jugendmedizin,

UKGM, Standort Gießen / Justus-Liebig-Universität, Gießen

\title{
Highlights der Jahrestagung der Deutschen Gesellschaft für Kinder- und Jugendmedizin 2015
}

\section{Ganzheitliche Gesundheitsforschung und -versorgung}

Sehr geehrte Kolleginnen und Kollegen,

vom 02. bis 05.09.2015 wird im Internationalen Congress Center München (ICM) die 111. Jahrestagung der Deutschen Gesellschaft für Kinder- und Jugendmedizin (DGKJ) stattfinden, zusammen mit der Deutschen Gesellschaft für Sozialpädiatrie und Jugendmedizin (DGSPJ), der Deutschen Gesellschaft für Kinderchirurgie (DGKCH) und dem Berufsverband Kinderkrankenpflege Deutschland (BeKD).

Da die Universitätskinderklinik Gießen bereits 1912 eröffnete wurde, aber noch keine Jahrestagung in der 132-jährigen Geschichte der DGKJ ausgerichtete hat, ist es für uns eine große Ehre, die diesjährige Tagung in München zu gestalten. Neben der „Suppe für den Säugling“ (1864) von J. Liebig, die den Beginn der künstlichen Säuglingsernährung darstellte, dem „Ramogen“ (1874) von P. Biedert, einem Gründungsmitglied der Deutschen Gesellschaft für Kinderheilkunde, der Erstbeschreibung der Ringelröteln (1899) durch G. Sticker und der Begründung der Pharmakokinetik (1953) durch F.H. Dost gingen bedeutsame Anregungen für die Kinder- und Jugendmedizin von Gießen aus. So setzte sich H.I. Koeppe, der erste Gießener Pädiater, ein Heubner-Schüler aus Leipziger Tagen und ärztlicher Leiter der „Zentrale für Mütter- und Säuglingsfürsorge im Großherzogtum Hessen“, bereits 1905 in Münchener Medizinische Wochenzeitschrift sehr entschie- den für die „lebensschwachen“ (untergewichtigen) Neugeborenen ein. Er verwies auf die Bedeutung der postnatalen Gesundheitsverhältnisse und argumentierte, dass „der [Kinder-] Überschuss abzunehmen beginnt, ein Säugling, die Kinder an Wert gewinnen und damit auch die Arbeit derer, die sich mit der Erhaltung der Kinder beschäftigen“. Dies ist ein Gesichtspunkt, der in der heutigen Diskussion über den Förderungsbedarf der Kinder- und Jugendmedizin im Hinblick auf die stagnierenden Geburtenraten und die Zunahme chronischer Erkrankungen zu kurz kommt. Koeppe erkannte auch die Bedeutung von Ernährung, Hygiene, Prävention, Mütter- und Schwangerenfürsorge sowie der Ausbildung von Ärzten und Pflegekräften zur Bekämpfung der Säuglingssterblichkeit.

Diesem umfassenden interdisziplinären Ansatz folgend wird die diesjährige Jahrestagung der DGKJ - vor dem Hintergrund einer zunehmend organbezogenen Medizin - die lebensalterbezogene Gesundheitsforschung und -versorgung im ganzheitlichen Sinne für die verschiedenen Akteure der Kinder- und Jugendmedizin in den Mittelpunkt stellen, um die Herausforderungen von Wissenschaft, Krankenversorgung, Sozialstaat und Ökonomie zu bestehen. Auch in München richtet sich das Tagungsprogramm an Kollegen vor, in und nach der Facharztausbildung zum Kinder- und Jugendmediziner, Studenten der Medizin, Kranken- pfleger, Psychologen, Sozialarbeiter, Pädagogen, Diätassistenten, experimentelle und klinische Forscher mit akademischen Ambitionen, an niedergelassene und leitende Kollegen der Kinderkliniken - aber ebenso an alle, die fachübergreifend mit dem komplexen Thema Kindergesundheit beschäftigt sind. Mithilfe von mehr als 25 pädiatrischen Fachgesellschaften und des Berufsverbands der Kinder- und Jugendärzte e. V. (BVKJ) sind 65 Workshops und 125 Symposien zusammengestellt worden, deren inhaltliche Schwerpunkte in der interdisziplinären Vermittlung von praktischem Wissen mit Fortund Weiterbildungscharakter bestehen. Auch werden diesmal „Updates“ der verschiedenen Disziplinen der Kinder- und Jugendmedizin angeboten.

》) Praktisches Wissen vermitteln 65 Workshops und 125 Symposien

Was ist bei der Tagung in München neu? Was macht sie als bedeutendste Fachtagung für Kindergesundheit in Deutschland zusätzlich attraktiv?

Wir greifen in München zahlreiche Anregungen der letzten Jahre auf und haben eine neue Tagungsstruktur festgelegt. Die Workshops finden nur am Mittwoch, den 02.09.2015, statt. Der Sonntag steht zur Entspannung im sommerlichen München zur Verfügung. Die Symposien starten jeden Morgen mit einer Plenarsitzung 
und einer „keynote lecture“ $z u$ den jeweiligen Hauptthemen der Tagung (Donnerstag: Ernährung, Freitag: Immunsuppression, Samstag: multiresistente Keime), die interdisziplinär gestaltet sind und ohne Parallelsitzungen stattfinden. Die Pausen zwischen den Sitzungen wurden auf eine halbe Stunde erweitert, um die Gelegenheiten für den persönlichen und den fachlichen Austausch sowie Kommunikationsmöglichkeiten mit Firmenvertretern zu verbessern. Die offizielle Kongresseröffnung findet am Donnerstag um 11.15 Uhr statt, die Mitgliederversammlung am Donnerstag um 16.45 Uhr.

Leitlinienbasiertes Wissen steht wie gewohnt in den Workshops und den Symposien im Vordergrund. Neben Klassikern wie Impfen, Vorsorgeuntersuchungen, Kindernotfällen u. a. werden neue Themen angeboten (http://www.dgkj2015. de). So werden in einem Symposium kurz vor der Veröffentlichung durch die DGKJ befindliche Leitlinien vorgesellt.

Für den akademischen Nachwuchs werden ganztätige englischsprachige Symposien mit internationalen Wissenschaftlern zur Molekularbiologie des Darms und der Lungen angeboten; dies sind wissenschaftliche Interessengebiete, die in Gießen und München schwerpunktmäßig vertreten sind. Ferner wurden die Möglichkeiten der Abstract-Einreichung erweitert, in dem auch bereits international bzw. auf speziellen Fachtagungen vorgestellte Studien eingereicht werden könnten. Abstracts mit aktuellen Studien, die thematisch in entsprechende Symposien passen, werden dort in Form von Kurzvorträgen der jungen Kollegen integriert. Zur Vertiefung wissenschaftlicher Arbeitstechniken wird ein Workshop wissenschaftlicher Methoden angeboten.

Für die Studenten wurde das Programm ausgeweitet, $u$. a. werden nicht nur Workshops für laufende Doktorarbeiten und Prüfungsthemen des Staatsexamens, sondern auch ein Ethik-Seminar angeboten.

Die interdisziplinäre Verknüpfung zeigt sich nicht nur mit der Kinderchirurgie und Sozialpädiatrie, sondern auch mit anderen Fachgesellschaften u. a. bei folgenden Themen: Kinderradiologie, Kinderorthopädie, frühe Hilfen, pädiatrische Epidemiologie, Kurzeingriffe in der Pädiatrie, perinatales Management von Kindern mit Fehlbildungen, Kurzdarmsyndrom und Rehabilitation. Darüber hinaus beteiligen sich am Tagungsprogramm neben zahlreichen Konventgesellschaften u. a. das Deutsche Jugendinstitut (DJI, München), die Deutsche Gesellschaft für Krankenhaushygiene (DGKH), die Deutsche Gesellschaft für Rechtsmedizin (DGRM), das Bayerische Landesamt für Gesundheit und Lebensmittelsicherheit (LGL), die Gesellschaft für Hygiene, Umweltmedizin und Prävention (GHUP) sowie die Ständige Impfkommission (STIKO) des Robert Koch-Instituts.

Die Kinderkrankenpflege ist zusätzlich zu den eigenen Sitzungen durch viele Themen in interdisziplinären Symposien verankert: u. a. Kinderschutz, psychosoziale Betreuung auf neonatologischen Intensivstationen, Fütterungsstörungen, Spiritualität und Religiosität in der Pädiatrie.

Es war diesmal ebenfalls ein Anliegen, eine Tagung auf der Basis von Teilnahmegebühren zu organisieren, die gerade für junge Pädiater und Studenten attraktiv sind. Dies wird auch durch den nichtselbstverständlichen Verzicht der Referenten auf Honorare ermöglicht und stellt letztlich ein Zeichen der Nachwuchsförderung sowie der Einheit in der Kinderund Jugendmedizin dar.

\section{》) Sozialpolitische Aspekte der Kinder- und Jugendmedizin thematisieren}

Neben dem inhaltlichen Programm für Fort-/Weiterbildung und Wissenschaft setzen wir auch diesmal öffentliche „Highlights", mit denen wir öffentlichkeitswirksam die sozialpolitischen Aspekte der Kinder- und Jugendmedizin zusammen mit Vertretern der Politik und des öffentlichen Lebens thematisieren. Erstmalig wurden mit den Ministerpräsidenten Seehofer und Bouffier Schirmherren für die DGKJ-Tagung gewonnen. Es gibt insgesamt 4 öffentliche Symposien:

1. Was können wir von der Pädiatrie in anderen Ländern lernen?

- Fünf in Deutschland ausgebildete Kollegen, die in ausländischen Kinderkliniken (Kanada, USA, Finn- land, England, Schweiz) eine führende Position einnehmen, beantworten unsere Fragen.

2. Das besondere Symposium „Du sollst dir kein Bildnis machen": von der Unmessbarkeit der Kinder

- Themen: Kindliche Bildungswege und Familie (W.E. Fthenakis, München), Vom Jäger und Sammler und echten Ernährungsproblemen (H. Daniel, München), Neuroenhancement (W. Graf, Yale).

3. Projekt Kinder-/Jugendmedizin: last, but not least rechtliche und ethische Rahmenbedingungen

- Themen: universitäre Pädiatrie; Grenzen der „medizinischen Selbstverwaltung" und des Wettbewerbs; Kinderschutz und Familie im Wandel; der schwedische Kinderbeauftragte: ein Erfolgsmodell; der moderne Experte: Legitimationsbeschaffer oder unabhängiger Wissenschaftler?

4. "What about the parents power?" (Wie steht es um die Macht der Eltern?): Eltern-/Selbsthilfe

- Themen u. a.: Stimmen die Rahmenbedingungen für eine kindgerechte Krankenversorgung? Was kann der Beitrag der Eltern (aber auch der Krankenkassen und der Kinderärzte) als Interessenvertreter erkrankter Kinder sein?

In diesem Jahr wird das Orchester der Deutschen Kinderärzte ein Benefizkonzert im Gasteig geben, in dem Platz für alle Kongressteilnehmer, aber auch für Münchener Bürger bereitsteht. Gespielt werden „Eroica“, „Mein Vaterland“ und „Die Moldau“.

Nicht zuletzt ist es uns ein zentrales Anliegen, eine Tagung zu organisieren, bei der durch die aktive Teilnahme von Kindern und ihren Eltern gezeigt wird, dass wir unsere „Mission“ primär als eine Tagung für Kinder und Jugendliche verstehen. So werden der Chor und die Big-Band der Liebig-Schule Gießen bei der Kongresseröffnung bzw. der Kongressparty auftreten. Letztere findet am Freitagabend, den 04.09.2015, nach dem Konzert des Kinderärzte-Orches- 
ters in der Muffathalle statt, die fußläufig zum Gasteig liegt. Derzeit läuft der Kunstwettbewerb „Essen in Hessen“ an hessischen Schulen, in dessen Rahmen Schüler zu bestimmten Themen der Ernährung künstlerische Ausdrucksformen entwickeln, die in einer digitalen Kunstausstellung im Foyer des ICM gezeigt werden. Auch beim Koch-Event im Rahmen des "get together" (Donnerstagabend, 03.09.2015) im Foyer des ICM werden Münchener Kinder zusammen mit prominenten Köchen und Politikern (türkisches Kochen, glutenfreies Kochen, Bekochen von Kindern mit Phenylketonurie) ihre Freude im kulinarischen Umgang mit Grundnahrungsmitteln demonstrieren. „Last but not least“ findet bereits am Mittwochabend (02.09.2015) ein Benefizfußballspiel mit Exspielern des FC Bayern München und Galatasaray Istanbul sowie chronisch kranken Kindern mit und ohne Migrationshintergrund statt. Hierbei wird die Notwendigkeit der guten medizinischen Versorgung als Voraussetzung für körperliche und psychosoziale Leistungsfähigkeit chronisch kranker Kinder thematisiert.

Wir danken bereits jetzt für die zahlreichen Anregungen, das starke Interesse an aktiver Teilnahme und die sehr konstruktiven Unterstützungen, die wir im Vorfeld der 111. DGKJ-Tagung erfahren haben. Wir hoffen, dass alle der Kindergesundheit verpflichteten Kollegen und Mitarbeitern bei dieser Tagung in München zahlreiche und innovative Impulse sowie vielfältige Gemeinsamkeiten für ihre weitere Arbeit mit Kindern und Jugendlichen entdecken.

\section{Geter ginmer}

Prof. Dr. K.-P. Zimmer

Kongresspräsident der DGKJ, München, 02.-05.09.2015

\section{Korrespondenzadresse}

Prof. Dr. K.-P. Zimmer

Abt. Allgemeine Pädiatrie \& Neonatologie,

Zentrum für Kinderheilkunde und

Jugendmedizin, UKGM,

Standort Gießen / Justus-Liebig-Universität

Feulgenstr. 12, 35392 Gießen

klaus-peter.zimmer@paediat.

med.uni-giessen.de

Interessenkonflikt. K.-P. Zimmer gibt an, dass kein Interessenkonflikt besteht.

\section{Wissenschaftsrat stärkt wissenschaftliche Integrität}

Wissenschaftliche Integrität ist die Grundlage für eine hohe Qualität und die Leistungsfähigkeit des Standorts Deutschland. Der Wissenschaftsrat hat diesbezüglich in einem Positionspapier Empfehlungen zur wissenschaftlichen Integrität verabschiedet, womit der Fokus zu einer umfassenden Kultur der Redlichkeit und Qualität an wissenschaftlichen Einrichtungen erweitert werden soll. Zu den Rahmenbedingungen der Integrität sollen vor allem die Vermittlung guter wissenschaftlicher Praxis, Beratung und Aufklärungsstrukturen in Konfliktfällen sowie eine stärkere Ausrichtung auf Qualität statt Quantität in der gesamten Forschungs- und Publikationspraxis zählen. Die Empfehlungen des Wissenschaftsrates widmen sich nicht nur gravierenden Fällen des Betrugs, sondern betrachten auch die Grauzone nicht integren Verhaltens. Damit geht der Wissenschaftsrat über den Schwerpunkt von Plagiatsfällen in Doktorarbeiten hinaus. Das Positionspapier beleuchtet Rahmenbedingungen, die wissenschaftliche Integrität erschweren können, wie etwa der Druck für Wissenschaftlerinnen und Wissenschaftler, möglichst viel und in hochrangigen Fachzeitschriften zu veröffentlichen, gepaart mit schwierigen Arbeitsbedingungen sowie starken Abhängigkeiten. Hierbei entstehe insbesondere für den Nachwuchs eine Situation, die integres Handeln gefährden kann. Laut Wissenschaftsrat sollen Hochschulen und wissenschaftliche Einrichtungen künftig im Umgang mit Verdachtsfällen spezielle Beratung in einer neu zu etablierenden, institutionenübergreifenden Einrichtung erhalten.

Quelle: Wissenschaftsrat, www.wissenschaftsrat.de 


\section{Hier steht eine Anzeige.}

黛 Springer 